\title{
Seizure activity causing loss of cardiac output after a Fontan operation
}

\author{
Sheila Lane, Piers Daubeney, Kevin Walsh, Keith Dawkins, Anthony Salmon
}

\begin{abstract}
After a Fontan operation pulmonary blood flow is heavily dependent on respiration. Two patients with a Fontan and normal sinus rhythm developed episodes of collapse with loss of cardiac output secondary to the tonic phase of an epileptic fit. The forced expiratory effort during the tonic phase severely compromised systemic venous return and led to collapse due to loss of cardiac output.

(Arch Dis Child 1995; 72: 62-63)
\end{abstract}

Keywords: Fontan, arrhythmia, epileptic fits.

The Fontan operation is performed as palliation for congenital heart disease when there is only one functional ventricle. ${ }^{1}$ The right atrium is anastomosed to the pulmonary artery so that pulmonary blood flow is largely dependent on a normal respiratory pattern with intermittent negative intrathoracic pressures. ${ }^{23}$

There is a high incidence of late arrhythmias after the Fontan operation and Stokes-Adams attacks and sudden death have been attributed to sinus node disease, atrial and ventricular dysrhythmias. ${ }^{4}$ We present two children with Fontan circuits who presented with episodes of collapse associated with sudden loss of cardiac output that were found to be precipitated by the tonic phase of epileptic seizures.

\section{Case reports}

CASE 1

An 8 year old boy presented with four episodes of collapse at home four years after a Fontan procedure for complex congenital heart disease. The parents reported cyanosis, absence of peripheral pulses and no response to mouth to mouth resuscitation. The episodes lasted for several minutes and then spontaneously resolved, with complete recovery by the time of admission to hospital.

Initial investigations included full blood count, serum electrolytes, glucose, liver function tests, a 24 hour electrocardiogram (ECG) and an electroencephalogram (EEG) which were all normal. Echocardiography and cardiac catheterisation showed the Fontan circuit was anatomically and functionally intact. Electrophysiological studies elicited atrial flutter with $1: 1$ and 2:1 conduction and it was assumed that the rapid ventricular response during episodes of atrial flutter had led to the loss of cardiac output and collapse. However, despite treatment with amiodarone and adequate plasma concentrations he con- tinued to collapse. An atrial pacemaker was implanted empirically to prevent possible episodes of sinus bradycardia or arrest. However, syncopal episodes continued and he was admitted for further investigation and monitoring. Fortuitously an episode was witnessed by medical staff when, after an expletive he became rigid, apnoeic, cyanosed, and collapsed with no palpable pulses. Continuous ECG monitoring during the episode showed sinus rhythm up to 120 beats per minute. Bag and mask ventilation failed to achieve chest expansion due to excessive respiratory muscle tone and external cardiac massage failed to produce output. After two minutes the tonic activity disappeared spontaneously, normal breathing and cardiac output returned, and the heart rate returned to about 80 beats per minute. After 30-40 minutes of drowsiness he fully recovered.

In the 24 hours before this episode he had EEG monitoring which continued for a further 48 hours after the attack. In EEG recordings both before and after collapse there was a clear epileptic focus in the left temporal region. After treatment with carbamazepine there have been no further collapses during 12 months' follow up.

\section{CASE 2}

An 8 year old girl had 13 episodes of collapse over a period of 18 months. She had a Fontan procedure four years before for tricuspid atresia with no complications. In each attack she felt nauseated and became pale, rigid, and apnoeic and then deeply cyanosed and collapsed. She would remain cyanosed and poorly perfused for several minutes before spontaneously recovering. There was no clonic activity. She would then be drowsy and unrousable for a long period before recovering fully.

Initial investigations revealed normal full blood count, serum electrolytes, glucose, and liver function tests. An echocardiogram suggested her Fontan was anatomically and functionally intact. EEG recordings showed excessive slow wave activity over the right temporoparietal region but no evidence of an epileptic focus. Twelve lead ECG suggested pre-excitation and therefore cardiac catheterisation and electrophysiology were performed. The Fontan circuit was satisfactory and no tachycardias were provoked by electrophysiology. Normal conduction intervals and recovery times were also demonstrated. Subsequent exercise testing, cranial computed tomography, and 24 hour ECG were all normal. 
In view of the lack of evidence for an arrhythmia a therapeutic trial of anticonvulsants (carbamazepine $15 \mathrm{mg} / \mathrm{kg} /$ day) was started. Since then there have been no further collapses and she remains well, despite some recent evidence of developmental delay.

\section{Discussion}

The Fontan procedure is carried out in cases of complex congenital heart disease where there is only one functional ventricle. ${ }^{1}$ The systemic venous return enters the pulmonary circuit via the right atrium and the right ventricle does not contribute to pulmonary blood flow. It has been proposed that in a Fontan circuit the blood flow to the lungs (and hence the cardiac output) is dependent upon inspiration ${ }^{2} 3$ (negative intrathoracic pressure), and atrial and systemic ventricular contraction. ${ }^{5}$

Arrhythmias and epileptic fits are recognised complications after cardiac surgery. ${ }^{6}$ Collapse with loss of cardiac output in such patients is usually attributed to an arrhythmia as loss of cardiac output is not a feature of an epileptic fit in a patient with an anatomically normal circulation. In the presence of the unique haemodynamics of a Fontan circulation, however, the raised intrathoracic pressure associated with the tonic phase of an epileptic fit appeared to have had a profound effect on cardiac output in the two patients described. During this phase of a generalised seizure the diaphragm and intercostal muscles become rigid and there is forced expiration followed by apnoea. We suggest that the loss of normal respiratory function and sudden increase in intrathoracic pressure severely compromises pulmonary blood flow leading to a sudden fall in cardiac output and collapse. A similar but less dramatic effect of raised intrathoracic pressure on circulation can be observed in patients with a normal circulation following a Valsalva manoeuvre. ${ }^{7}$

In our two cases, arrhythmias were initially suspected to be the cause of the syncopal episodes. In the first case the child had a pacemaker inserted and was treated with amiodarone to prevent potential tachycardia and bradycardia. When a syncopal episode was eventually witnessed in hospital a continuous ECG recording showed sinus rhythm throughout. EEGs before and after collapse clearly showed epileptic activity and there have been no further episodes since starting carbamazepine. In the second case carbamazepine was started on clinical grounds after full investigations had failed to elicit any arrhythmia and, as with case 1 , this abolished the syncopal episodes.

We believe that these two cases illustrate the severe clinical effects that seizures may have on the haemodynamics after a Fontan operation. Awareness of seizures as a possible cause of collapse with loss of cardiac output in these patients may prevent delay in diagnosis and treatment and thus prevent unnecessary morbidity.

1 Fontan F, Mounicot FB, Baudet E, Simonneau J, Gordo J, Gouffrant JM. 'Correction' de l'atresie tricuspidienne. Rapport de deux cas 'corriges' par l'utilisation d'une technique chirurgicale nouvelle. Ann Chir Thorac Cardiovasc 1971; 10: 39-47.

2 Williams DB, Kiernan PD, Metke MP, Marsh HM, Danielson MD. Haemodynamic response to positive endexpiratory pressure following right atrium-pulmonary expiratory pressure following right atrium-pulmonary
artery bypass (Fontan procedure). f Thorac Cardiovasc Surg artery bypass (Fon
1984; 87: $856-61$.

3 Penny DJ, Redington AN. Doppler echocardiographic evaluation of pulmonary blood flow after the Fontan operation: the role of the lungs. Br Heart $\mathcal{F} 1991$; 66: 372-4.

4 Chen SC, Nouvi S, Pennington DG. Dysrhythmia after modified Fontan procedure. Pediatr Cardiol 1988; 9: 215-9.

5 Qureshi SA, Richheimer R, McKay R, Arnold R. Doppler echocardiographic evaluation of pulmonary artery flow after modified Fontan operation: importance of atrial contraction. Br Heart $\mathcal{f} 1990 ; 64: 272-6$.

6 Ehyai A, Fenichel GM, Bender HW. Incidence and prognosis of seizures in infants after cardiac surgery with profound hypothermia and circulatory arrest. $\mathscr{f} A M A$ 1984; 252: 3165-7.

7 Goldberg H, Elisberg EI, Katz LN. The effects of the Valsalva-like manoeuvre upon the circulation in normal individuals and patients with mitral stenosis. Circulation 1952; 5: 38-47. 